\title{
A Novel Agent-Based User-Network Communication Model in Wireless Sensor Networks
}

\author{
Sang-Sik Kim and Ae-Soon Park \\ Electronics and Telecommunications Research Institute \\ \{pstring, aspark\}@etri.re.kr
}

\begin{abstract}
Wireless sensor networks generally have three kinds of objects: sensor nodes, sinks, and users that send queries and receive data via the sinks. In addition, the user and the sinks are mostly connected to each other by infrastructure networks. The users, however, should receive the data from the sinks through multi-hop communications between disseminating sensor nodes if such users move into the sensor networks without infrastructure networks. To support mobile users, previous work has studied various user mobility models. Nevertheless, such approaches are not compatible with the existing data-centric routing algorithms, and it is difficult for the mobile users to gather data efficiently from sensor nodes due to their mobility. To improve the shortcomings, we propose a view of mobility and propose a model to support a user mobility that is independent of sinks. The proposed model, finally, is evaluated by simulation of delivery ratio, latency, and network lifetime.
\end{abstract}

Keywords: User Mobility Support, Wireless Sensor Networks.

\section{Introduction}

Wireless sensor networks typically consist of three objects, as shown in Fig. 1: user, sink, and sensor node [1].

Firstly, a user is an object that disseminates an interest in the sensor field and collects data about the interest from sensor nodes. Secondly, a sink is an object that collects data. The sink receives an interest from a user and disseminates the interest inside sensor fields. The sink receives sensing data from sensor nodes and forwards the sensing data to the user. Lastly, a sensor node is an object that generates data about the interest and delivers the data to a sink.

As shown in Fig. 1, the user and the sinks are mostly connected to each other by infrastructure networks. The users, however, should receive the data from the sinks through multi-hop communications between sensor nodes if such users move around the sensor networks without infrastructure networks. Recently, applications transmitting data to moving users inside sensor fields, such as rescue in a disaster area or maneuvers in a war zone, have been on the rise in large-scale sensor networks [5]. (Firefighters and soldiers are users gathering data from sensor networks.)

To support mobile users in wireless sensor network, previous work has studied various user mobility models. But, until now, only three models supported the mobility 
of users for those applications: the direct user-network communication model, the GPS-based user-network communication model, and the topology-control-based usernetwork communication model.

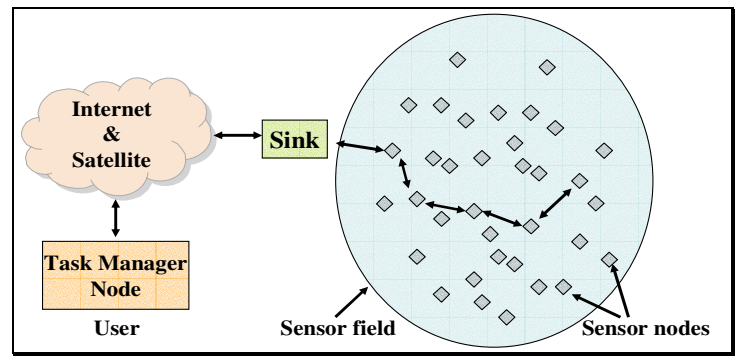

Fig. 1. Traditional Wireless Sensor Networks Model

The direct user-network communication model (D-COM) is shown in Fig. 2. It supports the mobility of a user on the assumption that the user communicates directly with sinks through infrastructure networks, namely, the Internet, such as communication systems in traditional sensor networks [1]. And, users can communicate directly with the networks via the sinks. But, in applications such as rescues in a disaster area or maneuvers in a war zone, circumstances without infrastructure networks, except sensor networks, are more prevalent. Hence, the assumption that a user and a sink can communicate directly through the Internet is not entirely accurate.

The GPS-based user-network communication model (G-COM) is seen in Fig. 3. G-COM is source-based topology [5], [6], [7]. In G-COM, sensor nodes proactively construct a GRID system with GPS receivers. G-COM assumes that all sensor nodes have their own GPS receivers and an ability that constructs a GRID when a stimulus is detected. A sensor node, i.e. source, with a stimulus is going to make a GRID in a sensor field. Once a GRID is set up, mobile user floods its interests within a cell only where the user is located. When a sensor node on a GRID receives interests, it sends interests to the source along a GRID path and data from the source are forwarded to the user along the reverse path.

The topology-control-based user-network communication model (T-COM) is seen in Fig. 4. It also identifies a user with a sink. This model supports the mobility of the user by reflecting the movement of the user [8], [9]. In T-COM, the user and sensor nodes construct a tree that is rooted at the user. The user always maintains the tree and gathers data from sensor nodes.

Intuitively, G-COM and T-COM seem to be suitable for the aforementioned applications. But, these models also have various problems. First of all, they cannot use existing effective data collection algorithms [2], [3], [4] between a sink and sensor nodes based on the data in static sink sensor networks because of low protocol compatibility. Accordingly, such algorithms can hardly be exploited if users in sensor networks have mobility. 


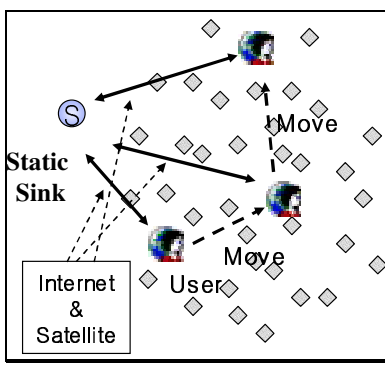

Fig. 2. Direct user-network communication model

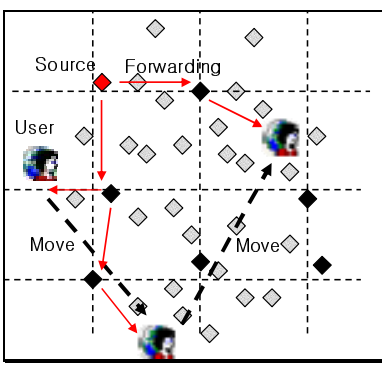

Fig. 3. GPS based usernetwork communication model

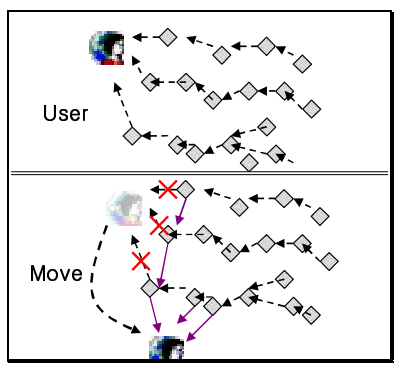

Fig. 4. Topology control based user- network communication model

The other problem is that the cost of the overhead to reorganize the network topology and reconstruct dissemination paths from sensor nodes to the mobile user is expensive. In G-COM, all sensor nodes make the topology based on location information. Accordingly, each sensor node must have its own GPS receiver. The cost of GPS receivers is decreasing, but the overall cost is still high. In T-COM, similarly, user mobility causes topology reconstruction. Users in T-COM have a tree that is rooted at each mobile user. If users move into a new location, then the root of trees must be changed, as seen in Fig. 4. This leads to enormous overhead to sensor nodes with constrained energy.

Hence, this paper proposes a novel agent-based user-network communication model (A-COM). A-COM collects data through a temporary agent and delivers the data to mobile users. In A-COM, if a user intends to obtain data while moving, the user appoints a sensor node to act as an agent, and the agent forwards interests to the sink via a sensor network. The sink, having received interests from the user, collects data from sensor nodes using the existing data collection algorithm in static sink sensor networks [2], [3], [4]. The collected data are finally forwarded to the user. (If there is no sink, the agent directly disseminates interests to the whole networks and collects data.)

Table 1. Taxonomy of Mobility Type

\begin{tabular}{ccccccc}
\hline $\begin{array}{c}\text { Mobility } \\
\text { Type }\end{array}$ & $\begin{array}{c}\text { Compatibility } \\
\text { with Existing } \\
\text { Static Sink } \\
\text { Routing Protocols }\end{array}$ & Feasibility & $\begin{array}{c}\text { GPS } \\
\text { receivers } \\
\text { for sensors }\end{array}$ & $\begin{array}{c}\text { Control } \\
\text { Overheads } \\
\text { according to } \\
\text { user mobility }\end{array}$ & $\begin{array}{c}\text { Control } \\
\text { Overheads to } \\
\text { support } \\
\text { multiple users }\end{array}$ & $\begin{array}{c}\text { Help of } \\
\text { infrastructure } \\
\text { networks }\end{array}$ \\
\hline D-COM & High & Low & Needless & Low & Low & Mandatory \\
G-COM & Low & Middle & Mandatory & Middle & Low & Needless \\
T-COM & Low & High & Needless & High & High & Needless \\
A-COM & High & High & Needless & Low & Low & Needless \\
\hline
\end{tabular}

A-COM has various advantages, as can be seen in Table 1. First of all, A-COM has the compatibility with existing static sink routing protocols without infrastructure networks. In addition, the users in A-COM do not make a topology (tree or GRID) and communicate only with agents. So, the users, while moving, are free from 
topology control. The user's freedom saves much energy, and enables more users to participate in the proposed model even if the sensors have no GPS receivers.

Our simulation verifies that the lifetime of sensor networks is prolonged because the user's freedom decreases the energy consumption of the sensor nodes. Also, we verified that the performance of the data delivery ratio and the delay never decreases. Nevertheless, the movement of the user is supported by only sensor nodes without infrastructure networks.

The rest of this paper is organized as follows. Section 2 describes the proposed model. Simulation results are presented in Section 3 to evaluate the effectiveness of our design and to analyze the impact of important parameters. Section 4 concludes the paper.

\section{Model Analysis}

In our model, if a user intends to obtain data while moving, the user appoints a sensor to act as an agent and forwards an interest to the agent. If there is one or more $\operatorname{sink}(\mathrm{s})$, the agent forwards interests to sensor networks via $\operatorname{sink}(\mathrm{s})$. The number of sinks, however, depends on the network policy. A network administrator might want to set a single or more sinks in the sensor field, or alternatively the sensor field may be hazardous as he cannot reach the field. Hence, we consider three scenarios according to the number of sinks and describe the scenarios based on following assumptions.

- A user can communicate with static sinks only through sensors because networks within sensor fields are infrastructure-less networks.

- It is possible that multiple sinks are deployed in sensor networks and are connected to each other via the Internet. How to connect a sink with other sinks is out of the range of this paper, but this helps maximize the efficiency of gathering data.

- Multiple static sinks are located in the outskirts of sensor fields.

- The data which one sink collects is aggregated by the sinks.

The aggregated data is shared by every multiple static sinks through the infrastructure network, namely, Internet.

- The interest from a user describes how many times the sink forwards the gathered data set to a user.

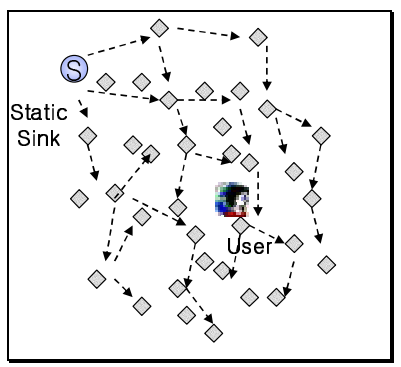

Fig. 5. Dissemination of Sink Announcement Message

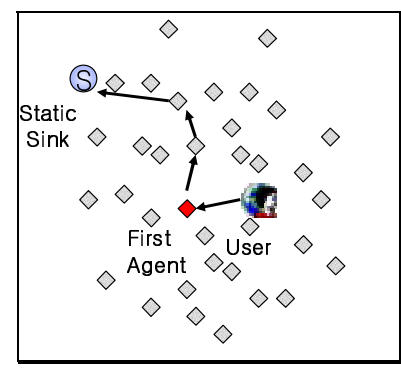

Fig. 6. Interest Dissemination of the user 


\subsection{Scenario 1: Sensor Fields with Only One Sink}

Dissemination of sink announcement message. In the initial stage of the sensor network, the network administrator sets a sink in a suitable position: center, outskirt, or a special position according to his or her policy. If a sink is located in an arbitrary position in sensor fields, it floods a sink announcement message to announce itself inside the whole sensor field (Fig. 5). As a result of the flooding announcement message, every sensor node knows the hop counts and next hop neighbor sensor node to the sink.

Dissemination of the user interest. While moving inside the sensor fields, if a user wants to collect data, the user selects the nearest node as a first agent, as shown in Fig. 6. The user delivers an interest to the first agent. The first agent, to which the user has delivered the interest, forwards the interest to the next hop neighbor node toward the sink. The next hop sensor node, which has had the interest delivered to it, also forwards the interest to the next hop neighbor node toward the sink. This process continues until the sink receives the interest of the user. Also, a route for the interest from the sink to the user has been established through this process. Based on our assumptions, the established route vanishes from the network when the described period in the interest is over.

Data collection of sink. A sensor network with a static sink is a network where sensing data from sensor nodes should be transmitted to the static sink through multihop communication. So, existing routing algorithms for a static sink can be used (e.g., routing algorithms collecting data by periods, routing algorithms collecting a minority event, or routing algorithms detecting a moving object.)

Hence, the network administrator can support a suitable routing protocol for users, and besides, a user can select and use the most appropriate routing algorithm with a static sink according to the policy of the networks. Such research has already been advanced [2], [3], [4]. So we will not mention it further in this paper. Rather, we use one of the existing routing algorithms to collect data in this paper. In Fig. 7, the static sink can forward interests from users to sensors and gather data from sensor networks according to the existing routing protocols. If all data are gathered by routing protocols, the static sink aggregates all data and forwards an aggregated data to the first agent.

Mobility support of the user. A user may move to another place after sending an interest to the first agent. In this case, the user selects another agent that can communicate with the first agent. Also, the user makes a new connection between the newly selected agent and the original agent. (While moving inside the sensor field, the user can make more agents and connections.) These agents and connections are used for forwarding the aggregated data from the sink.

Data propagation of sink. A sink delivers the aggregated data to the first agent through the reverse path of interest forwarding. The first agent delivers the aggregated data to the last agent through the connection of agents. The last agent delivers the 


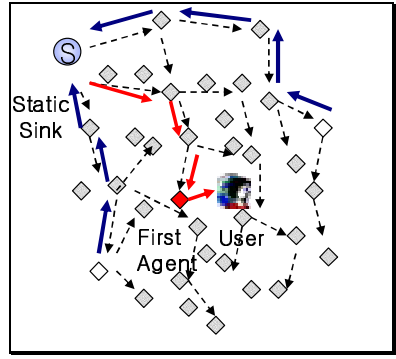

Fig. 7. Data Propagation to the user

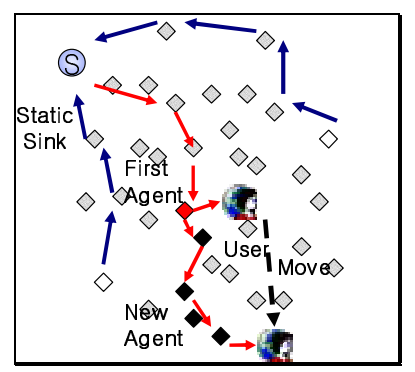

Fig. 8. Mobility support of the user

aggregated data to the user. As shown in Fig. 8, the user can surely receive the aggregated data from the sink.

\subsection{Scenario 2: Sensor Fields with Multiple Sinks}

Separation of the sensor fields. Basically, the difference between Scenario 1 and Scenario 2 is only the number of sinks. If there are more than one sink in the sensor field, this means a separation of the sensor fields. As a result of sink announcement message dissemination in this case, all sensor nodes know the nearest sink according to the hop counts. Accordingly, Interest dissemination of the user targets the nearest sink from the agent, as shown Fig. 9. The targeted sinks can be changed whenever the user wants to send its interests (see Fig. 10). Nevertheless, mobility support of the user and data propagation of the sink is still the same with Scenario 1. In addition, users may not be able to recognize how many static sinks are in the sensor fields. This means that the proposed model is independent of the number of sinks.

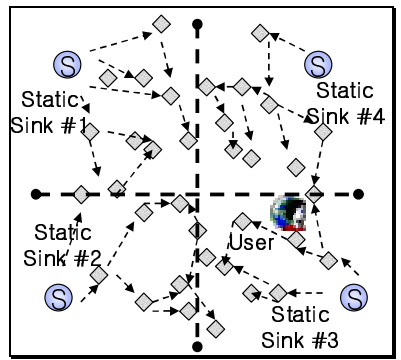

Fig. 9. Seperation of the Sensor Fields

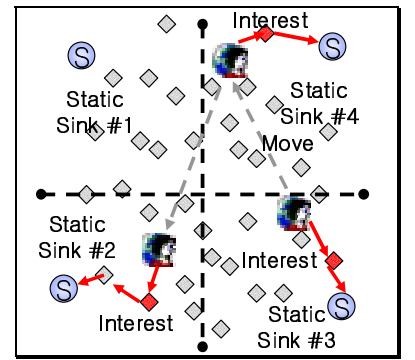

Fig. 10. Interest Dissemination of the user with multiple sinks

Data sharing of multiple static sinks. As shown in Fig. 1, a sink in typical sensor networks takes charge of the function as a gateway for a connection with infrastructure networks [1]. Various papers in relation to multiple static sinks also indicate the connection between a sink and an infrastructure network and the connection between all sinks as an assumption [10], [11]. 
Therefore, in this paper, it is assumed that each sink placed in the edge of a sensor field can communicate with the other sinks via the infrastructure networks. Hence, the proposed model of this paper collects data by one sink through sensor fields. And the aggregated data of the collected data will be shared by multiple static sinks through the infrastructure network.

Advantages with multiple static sinks. The proposed model can obtain various advantages with multiple static sinks. First of all, a user can receive the data from the nearest sink to its position. Therefore, short hops communications between a user and a sink are possible. This saves energy, enhances the data delivery ratio, and reduces delay. Also, because a user requires a dissemination of interests through multiple static sinks, the locations of data collection are diverse. It relieves the hot spot problem, which has carried a disproportionate amount of traffic to sensor nodes near the sink [13]. As a result, the lifetime of the sensor networks will increase because a balance of energy consumption of the sensor nodes is made possible.

\subsection{Scenario 3: Sensor Fields with No Sink}

Hazardous sensor fields. Sensor fields without a sink are a special type of sensor networks. If the sensor field is hazardous such that network administrator cannot reach the field (e.g., a battlefield), the sensor field may not have any sinks. In a battlefield, users (or soldiers) may move into a sensor field that has no sink and gather data from sensors. In this case, users must gather data for themselves.

Dissemination of sink announcement message. Because there is no sink in the sensor field, the sensor network cannot perform the sink announcement message dissemination process for itself. In this case, users appoint the nearest sensor node as first agent, and the first agent disseminates the sink announcement message. As shown in Figs. 11 and 12, users that want to gather data from sensors examine nearby sensor nodes whether there is a sink in the sensor field or not. If there is no sink, users appoint the nearest sensor node as first agent. Once a sensor node becomes the first agent, it acts as the sink of Scenario 1. Hence, other processes such as sink announcement message dissemination, interest dissemination of the user, mobility support of the user, and data propagation of the sink are the same as in Scenario 1.

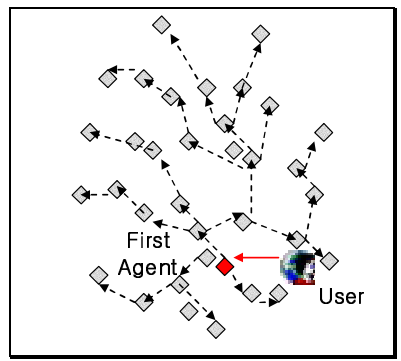

Fig. 11. First Agent Selection and Announcement

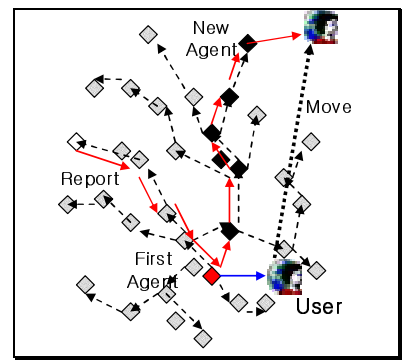

Fig. 12. Mobility support of the user 
Advantages with idle sensor network. Based on our assumptions, the proposed model in this scenario may create many first agents periodically. The first agents must return to the original state after the described period. This means that the first agents are appointed whenever users want to send their interests. Then, the first agents are reactively selected and perform all processes for user mobility. In the whole network, therefore, the sensor network can remain in an idle state in case there is no user in the sensor field. From the standpoint of the whole network, this is a positive effect because there is no control of messages and interests in the idle state sensor network.

\section{Performance Evaluation}

In this section, we evaluate the performance of a proposed model through simulations. We first describe our simulation model and simulation metrics. We next evaluate how environmental factors and control parameters affect the performance of a proposed algorithm.

\subsection{Simulation Model and Metric}

We evaluate the proposed model in Qualnet, a network simulator [12]. A sensor node's transmitting and receiving power consumption rate are $0.66 \mathrm{~W}$ and $0.39 \mathrm{~W}$. The transceiver in the simulation has a $50 \mathrm{~m}$ radio range in an outdoor area. Each interest packet is 40 bytes long, and the data packet has 64 bytes. The sensor network consists of 100 sensor nodes, which are randomly deployed in a 300m x 300m field.

We consider three scenarios for the proposed model according to the number of sinks. Hence, the number of sinks and users is changed for this evaluation. The multiple static sinks are located in the outskirts of sensor fields. And the user, which follows a random waypoint model of $10 \mathrm{~m} / \mathrm{s}$ speed and 10 second pause time, moves into the sensor field. The user disseminates an interest at an interval of every 10 seconds. Every sensor node receives the interest and generates only one sensing data for the interest. This is defined as one interest round. Namely, one interest round is 10 seconds. The simulation lasts for 500 seconds.

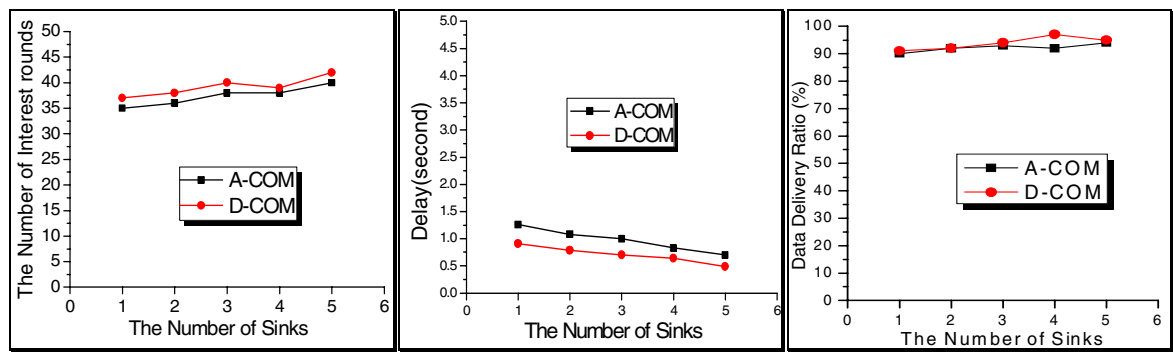

Fig. 13. Network lifetime for the Number of Sinks
Fig. 14. Delay for the Number of Sinks
Fig. 15. Data Delivery Ratio for the Number of Sinks 
We use metrics to evaluate the performance of the proposed algorithm. The network lifetime is defined as the number of the interest round when the first sensor node dies. The data delivery ratio is the ratio of the number of successfully received reports by a user to the total number of reports generated by every sensor node. The delay is defined as the average time between the time a user transmits an interest and the time the user receives the report. We compare three mobility types (D-COM, G-COM, and T-COM) in Table 1 with the proposed model in the simulation.

\subsection{Impact of the Number of Static Sinks}

Scenarios 1 and 2 of A-COM can be compared with the D-COM because G-COM and T-COM have no static sink. We first study the impact of the number of sinks on A-COM's performance. The number of sinks varies from 1, 2, 3, 4 to 5. And there is only one user in the sensor field. In this part, we compare Scenarios 1 and 2 to $\mathrm{D}-\mathrm{COM}$ regarding lifetime, delay, and delivery ratio.

A difference between A-COM and the D-COM is how to communicate between a user and a sink. Fig. 13 shows the number of interest rounds, namely, network lifetime. As shown in Fig. 13, the number of interest rounds shows little difference between A-COM and D-COM. This means that A-COM can manage sensor fields as well as D-COM without infrastructure that connects users with sinks. In addition, the lifetime is increased according to the number of sinks. This is a side effect of multiple sinks. Multiple sinks separate the sensor field, and besides, users only use the nearest sink to send interests and receive replies. Users can use the shortest path to communicate with multiple sinks. As a result of the shortest communication, the lifetime in A-COM is enhanced according to the number of sinks.

The delay is also enhanced by this side effect of multiple sinks. A-COM basically has some delay due to multi-hop communication between users and sinks. However, the delay is diminished according to the number of sinks, as shown in Fig. 14. Nevertheless, the data delivery ratio of A-COM is comparable with D-COM, as shown in Fig. 15. This also proves that the proposed model can manage sensor fields as well as D-COM without infrastructure.

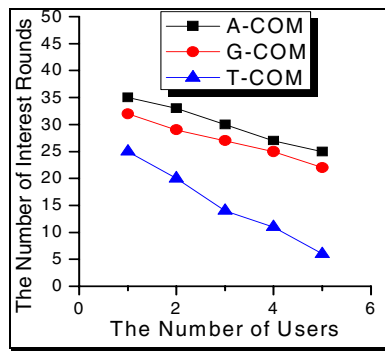

Fig. 16. Network Lifetime for the Number of Users
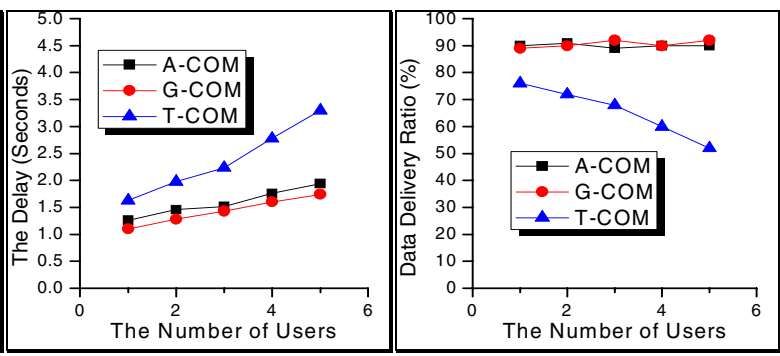

Fig. 17. Delay for the number of Users
Fig. 18. Data Delivery Ratio for the Number of Users 


\subsection{Impact of the Number of Users}

The number of users only results in path increase between users and sinks. D-COM uses direct communication between users and sinks, and A-COM uses multi-hop communication. A-COM has more paths and consumes more energy. (e.g., five users in A-COM consumes five times of the energy that is consumed by one user.) However, it is a tradeoff between energy and infrastructure. Although A-COM has more energy consumption and delays than D-COM, the merit of A-COM is infrastructure-less communication systems.

Scenario 3 of A-COM can be compared with G-COM and T-COM because Scenario 3 of A-COM, G-COM, and T-COM have no static sinks. There are no sinks, and the number of users varies from 1,2, 3, 4 to 5 . In this part, we compare Scenario 3 of A-COM to G-COM and T-COM regarding lifetime, delay, and delivery ratio.

G-COM and T-COM make and change the topology proactively, but Scenario 3 of A-COM reactively makes and shares it among users. Generally, users move about the sensor field only and generate its interest occasionally. Hence, sensors in Scenario 3 can save considerable energy. Alternatively, sensors in G-COM and T-COM maintain a topology continuously. Fig. 16 shows each lifetime of these sensor networks. As shown in Fig. 16, the lifetime of T-COM is considerably low due to frequent topology change and that of G-COM is relatively low due to GRID maintenance.

In Fig. 17, G-COM has little delay due to proactive GRID topology by the GPS receiver. T-COM proactively creates the topology, but frequent topology changes of TCOM delay data delivery considerably. The delay of Scenario 3, as shown in Fig. 17, however, is only a little high due to the reactive first agent selection and topology construction. In the case of the data delivery ratio, A-COM and G-COM in Fig. 18 are similar except for T-COM. The reason is frequent topology change. Topology change messages disturb the data delivery ratio.

\subsection{Impact of the Number of Sensor Nodes}

Evaluating A-COM with other models according to the number of sensors is of no real consequence. It is closely related to the performance of routing protocols that are used in the network. Hence, we do not evaluate the proposed model with this factor.

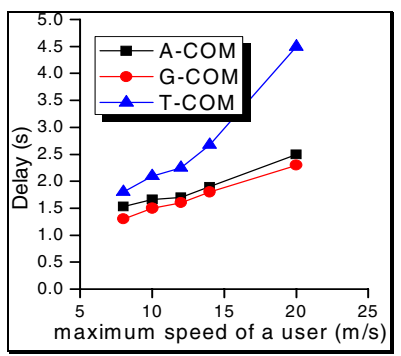

Fig. 19. Delay for User Speed

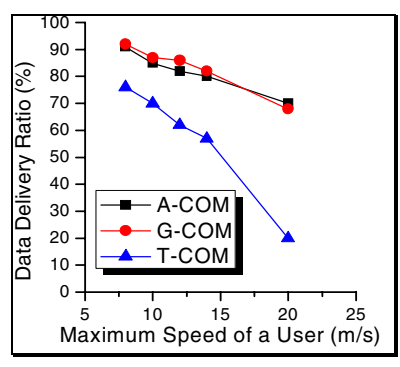

Fig. 20. Data Delivery Ratio for User Speed 


\subsection{Impact of the User Mobility}

We lastly evaluate the impact of user speed on A-COM. We vary the maximum speed of a user from $8,10,12,14$ to $20 \mathrm{~m} / \mathrm{s}$. We assume that there is one user in the sensor field. In this part, we compare Scenario 3 to G-COM and T-COM because D-COM is independent of user speed. Fig. 19 shows the delay in data delivery, which slightly increases as the user moves faster. The delay depends on a movement operation that is processed by the user. The faster a user moves, the more the time is needed to establish a connection between the user and the network. Nevertheless, the delay of A-COM is comparable with G-COM because A-COM creates only one communication path between the user and its first agent. The delay of T-COM, on the other hand, is relatively higher than the others due to frequent topology changes.

And, Fig. 20 shows the data delivery ratio when the user's moving speed changes. The data delivery ratio of A-COM is slightly decreased according to the delay. But the data delivery ratio remains around $0.8-0.9$; nevertheless, the user moves faster. Besides, the data delivery ratio of G-COM remains high because the GPS receiver may help the user with geographical routing. On the other hand, the data delivery ratio of T-COM is relatively lower than the others because it has too many topology changes when moving. The results in Fig. 19 and Fig 20 mean that A-COM is fast and stable without GPS receiver.

\section{Conclusion and Further Work}

In this paper, we propose a novel agent-based user-network communication model to support the mobility of users in wireless sensor networks. In the proposed network model, the user can receive data with a higher data delivery ratio and in a faster time without infrastructure.

We verified that the lifetime of sensor networks is prolonged because the reactive path construction decreases the consumption of sensor nodes. Also, we verified that performance of the data delivery ratio and the delay never falls; nevertheless, communication between the user and the network for guaranteeing movement of the user is supported by only sensor nodes without infrastructure networks.

There is further work that is related to this research. In a mobile environment, many sensor nodes can shift from one place to another frequently. The mobile sensor node environment makes more dynamic sensor networks. The issue of node mobility requires further study.

\section{References}

1. I.F. Akyildiz, et al., "A survey on sensor networks," IEEE Communications Magazine, Vol. 40, pp. 102-114, Aug. 2002.

2. C. Intanagonwiwat, et al., "Directed diffusion: A scalable and robust communication paradigm for sensor networks," ACM Mobicom, 2000.

3. C. Schurgers and M.B. Srivastava, "Energy efficient routing in wireless sensor networks," IEEE MILCOM 2001. 
4. W. R. Heinzelman, et al., "Adaptive Protocols for Information Dissemination in Wireless Sensor Networks," ACM Mobicom, 1999.

5. F. Ye, et al., "A Two-Tier Data Dissemination Model for Large-scale Wireless Sensor Networks," ACM MobiCOM, Sept. 2002.

6. S. Kim, et al., "SAFE: A Data Dissemination Protocol for Periodic Updates in Sensor Networks," Distributed Computing Systems Workshops 2003.

7. H. L. Xuan and S. Lee, "A Coordination-based Data Dissemination Protocol for Wireless Sensor Networks," IEEE ISSNIP, Dec. 2004.

8. K. Hwang, et al., "Dynamic sink oriented tree algorithm for efficient target tracking of multiple mobile sink users in wide sensor field," IEEE VTC, Sep. 2004.

9. S. R. Gandham, et al., "Energy Efficient Schemes for Wireless Sensor Networks with Multiple Mobile Base Stations," IEEE GLOBECOM, Dec. 2003.

10. H. Ferriere, et al., "Efficient and Practical Query Scoping in Sensor Networks," IEEE International Conference on Mobile Ad-hoc and Sensor Systems, Oct. 2004.

11. E. I. Oyman and C. Erso, "Multiple Sink Network Design Problem in Large Scale Wireless Sensor Networks," IEEE ICC, Jun. 2004.

12. Scalable Network Technologies, Qualnet, [online] available: http://www.scalablenetworks.com.

13. Hui Dai and Rechard Han, "A node-centric load balancing algorithm for wireless sensor networks,” IEEE GLOBECOM, Dec. 2003. 